\title{
Long non-coding RNA SNHG8 promotes autophagy as a ceRNA to upregulate ATG7 by sponging microRNA-588 in colorectal cancer
}

\author{
CHI HE ${ }^{1}$, YI FU ${ }^{2}$, YAN CHEN ${ }^{3}$ and XIQUAN $\mathrm{LI}^{1}$ \\ ${ }^{1}$ Department of General Surgery, Shenyang Anorectal Hospital, Shenyang, Liaoning 110054; \\ ${ }^{2}$ Department of General Surgery, The Second Hospital of Dalian Medical University, Dalian, Liaoning 116000; \\ ${ }^{3}$ Department of General Surgery, Daqing Oilfield General Hospital, Daqing, Heilongjiang 163000, P.R. China
}

Received October 11, 2020; Accepted February 4, 2021

DOI: 10.3892/ol.2021.12838

\begin{abstract}
Colorectal cancer (CRC) is the third most common cancer worldwide. Long non-coding RNA (lncRNA) small nucleolar RNA host gene 8 (SNHG8) acts as an oncogene in different types of cancer, including prostate, breast and ovarian cancer. SNHG8 promotes the tumorigenesis of CRC; however, its underlying molecular mechanism remains unclear. The present study aimed to explore the mechanism of SNHG8 on CRC development via various assays, including western blot, pull-down, PCR and immunofluorescence assays. The results of the present study demonstrated that SNHG8 expression was substantially upregulated in primary tumor tissues from The Cancer Genome Atlas dataset. Western blot and immunofluorescence analyses demonstrated that SNHG8 facilitated cell proliferation and autophagy in CRC cells. Notably, the function of SNHG8 in enhancing autophagy was dependent on autophagy-related gene 7 (ATG7). In addition, western blot analysis indicated that the effect of SNHG8 on autophagy in CRC cells was dependent on the miR-588/ATG7 axis. Taken together, the results of the present study suggest that SNHG8 promotes autophagy in CRC cells.
\end{abstract}

\section{Introduction}

Colorectal cancer (CRC) is the third most common cancer and the fourth most common cause of cancer-associated mortality

Correspondence to: Dr Xiquan Li, Department of General Surgery, Shenyang Anorectal Hospital, 9 Nanjingbei Street, Shenyang, Liaoning 110054, P.R. China

E-mail: shenyang_xiquan@126.com

Abbreviations: CRC, colorectal cancer; ATGs, autophagy-related genes; LC3, microtubule-associated protein 1 light chain 3B; lncRNA, long non-coding RNA; SNHG8, small nucleolar RNA host gene 8; NC, negative control; siRNA, small interfering RNA

Key words: SNHG8, autophagy, CRC, ATG7, microRNA-588 worldwide; it is estimated that $\sim 135,430$ new cases of colon cancer are diagnosed and 50,260 deaths occur each year in the United States of America $(1,2)$. Despite recent advancements in the therapeutic treatments for patients with CRC, the clinical outcome of this disease remains unsatisfactory (3). Thus, it is important to investigate the molecular mechanisms underlying the development of CRC to identify effective therapeutic targets.

Autophagy is a biological process involving several key genes named autophagy-related genes (ATGs), and is involved in cellular metabolism, which depends on the lysosomal degradation of cargo (viruses, bacteria and proteins) (4). The main role of autophagy is to eliminate misfolded proteins, organelles or other factors that cannot be degraded by the proteasome (4). Autophagy protects cells from stress and helps cells survive under stressed conditions (4). Previous studies have demonstrated that autophagy mediates CRC metastasis and proliferation $(5,6)$. Increasing the autophagy level promotes the growth of advanced cancer and enhances drug resistance of cells in different types of cancer (7). However, the molecular mechanisms underlying autophagy in CRC remain unclear.

Long non-coding RNAs (lncRNAs) are a class of non-protein coding RNAs $>200$ nucleotides in length (8). Previous studies have reported the unique association between lncRNAs and the development of CRC $(8,9)$. For example, it has been demonstrated that lncRNA small nucleolar RNA host gene 8 (SNHG8) mediates autophagy by upregulating autophagy-related gene 7 (ATG7) expression in hepatic ischaemia/reperfusion injury (10). In addition, IncRNA CDKN2B antisense RNA 1 promotes tumor growth and metastasis in hepatocellular carcinoma by upregulating the mRNA expression of nucleosome assembly protein 1-like 1 (11). Several studies have reported that the regulatory network of lncRNAs is both conventional and crucial $(8,9)$. SNHG8 is a novel lncRNA (12) that has been investigated in a number of studies $(10,12-14)$; however, its underlying molecular mechanisms in CRC progression and autophagy remain unclear.

MicroRNAs (miRNAs/miRs) are non-coding RNAs that regulate gene expression by altering the 3'-untranslated region (UTR) of specific genes, resulting in mRNA degradation (15). 
Increasing evidence suggest that miRNAs can mediate carcinogenesis by regulating tumor-related genes (16). For example, a study demonstrated that miR-588 expression is downregulated in lung cancer, and acts as a tumor suppressor that targets with progranulin (17). In addition, miR-588 has been reported to regulate breast cancer, CRC and gastric cancer $(18,19)$. However, the underlying molecular mechanisms of miR-588 in CRC remain unclear, particularly in autophagy. Thus, the present study aimed to investigate the role of miR-588 in CRC.

\section{Materials and methods}

Cell culture. Colorectal cell lines, HCT116, FHC, HCT8, HT29 and SW480, were purchased from the Cell Bank of Type Culture Collection of The Chinese Academy of Sciences. Cross-contamination of the cell lines was excluded via short tandem repeat profiling $(20,21)$. CRC cells were maintained in DMEM supplemented with $10 \%$ fetal bovine serum (both purchased from Gibco; Thermo Fisher Scientific, Inc.) and 1\% penicillin-streptomycin (Invitrogen; Thermo Fisher Scientific, Inc.), at $37^{\circ} \mathrm{C}$ with $5 \% \mathrm{CO}_{2}$.

Cell transfection. Plasmids, including SNHG8 cDNA and pcDNA-3.1 (5 $\mu \mathrm{g} / 1 \times 10^{4}$ cells), and miR-588 mimics and negative control (mimic-NC) $\left(3 \mu \mathrm{g} / 1 \times 10^{4}\right.$ cells) were purchased from Shanghai GeneChem Co., Ltd. Small interfering RNA (siRNA) and NC oligonucleotides of ATG7 were purchased from Sigma-Aldrich (Merck KGaA) ( $3 \mu \mathrm{g} / 1 \times 10^{4}$ cells). HCT116 and SW480 cells were transfected using Lipofectamine ${ }^{\circledR} 3000$ reagent (Invitrogen; Thermo Fisher Scientific, Inc.) for $6 \mathrm{~h}$ at $37^{\circ} \mathrm{C}$, according to the manufacturer's protocol. Subsequent experiments were performed $24 \mathrm{~h}$ after transfection. The following sequences were used: mimic-NC forward, 5'-UUC UCCGAACGUGUCACGUTT-3' and reverse, 5'-ACGUGA CACGUUCGGAGAATT-3'; mimic-miR-588 forward, 5'-GCU UCCAAAGAUCAGGUAACATT-3' and reverse, 5'-UGUUAC CUGAUCUUUGGAAGCTT-3'; si-ATG7 forward, 5'-CAG CCUGGCAUUUGAUAAATT-3' and reverse, 5'-UUUAUC AAAUGCCAGGCUGTT-3'; and si-NC forward, 5'-UUCUCC GAACGUGUCACGUTT-3' and reverse, 5'-ACGUGACAC GUUCGGAGAATT-3'.

Reverse transcription-quantitative $(R T-q) P C R$. Total RNA was extracted from HCT116 and SW480 cells using the TRIzol $^{\circledR}$ reagent kit (Invitrogen; Thermo Fisher Scientific, Inc.), according to the manufacturer's protocol. Total RNA concentration was detected using a NanoDrop spectrophotometer (ND-1,000; Thermo Fisher Scientific, Inc.), at a wavelength of $260 \mathrm{~nm}$. Total RNA $(0.5 \mu \mathrm{g})$ was reverse transcribed into cDNA using the PrimeScript RT reagent kit (Takara Biotechnology Co., Ltd.) at $25^{\circ} \mathrm{C}$ for $10 \mathrm{~min}, 48^{\circ} \mathrm{C}$ for $30 \mathrm{~min}$ and $95^{\circ} \mathrm{C}$ for $5 \mathrm{~min}$. qPCR was subsequently performed using the Power SYBR-Green PCR Master Mix (Thermo Fisher Scientific, Inc.), at a final volume of $10 \mu \mathrm{l}$, containing $0.04 \mu \mathrm{g}$ cDNA. U6 was used as the internal control for miR-588. The following thermocycling conditions were used: Initial denaturation at $95^{\circ} \mathrm{C}$ for $10 \mathrm{~min}$, followed by 40 cycles for $15 \mathrm{sec}$ at $95^{\circ} \mathrm{C}$ and $60^{\circ} \mathrm{C}$ for $1 \mathrm{~min}$. The subsequent melt curve stage consisted of $95^{\circ} \mathrm{C}$ for $15 \mathrm{sec}, 60^{\circ} \mathrm{C}$ for $1 \mathrm{~min}$ and $95^{\circ} \mathrm{C}$ for $15 \mathrm{sec}$. Relative expression levels were calculated using the $2^{-\Delta \Delta \mathrm{Cq}}$ method (22). The following primer sequences were used: SNHG8 forward, 5'-AAGTTTACAAGC ATGCGCGG-3' and reverse, 5'-TCAAACTGACGGTTCTCG GG-3'; ATG3 forward, 5'-GAGCGGCTCCTCAAGGAA-3' and reverse, 5'-TGTAGCCCATTGCCATGTTGG-3'; ATG5 forward, 5'-AAAGATGTGCTTCGAGATGTGT-3' and reverse, 5'-CACTTTGTCAGTTACCAACGTCA-3'; ATG7 forward, 5'-GAACAAGCAGCAAATGA-3' and reverse, 5'-GACAGAGGGCAGGATAG-3'; ATG10 forward, 5'-AAT GGAAGGGCGACAGTGAG-3' and reverse, 5'-AGTCCTACA CGCCACTTGAC-3'; ATG12 forward, 5'-CTGCTGGCGACA CCAAGAAA-3' and reverse, 5'-CGTGTTCGCTCTACTGCC C-3'; GAPDH forward, 5'-GACTCATGACCACAGTCCATG C-3' and reverse, 5'-AGAGGCAGGGATGATGTTCTG-3'; miR-588 forward, 5'-TACTCAACTCACTACTGCATGG-3' and reverse, 5'-TATCGAAGTTCTGCTCTCTGTC-3'; and U6 forward, 5'-CTCGCTTCGGCAGCACA-3' and reverse, 5'-AACGCTTCACGAATTTGCGT-3'.

Western blotting. Total protein was extracted from CRC cells (HCT116 and SW480) using RIPA lysis buffer (Cell Signaling Technology, Inc.). Protein concentration was quantified using the bicinchoninic acid assay kit (Beyotime Institute of Biotechnology), according to the manufacturer's protocol, and $5 \mu \mathrm{l}$ protein/lane was separated by $10 \%$ SDS-PAGE gels. The separated proteins were subsequently transferred onto PVDF membranes (Sigma-Aldrich; Merck $\mathrm{KGaA})$ and blocked with non-fat milk dissolved in TBST (10 mM Tris- $\mathrm{HCl}, \mathrm{pH} 7.4,150 \mathrm{mM} \mathrm{NaCl}, 0.1 \%$ Tween-20) for $2 \mathrm{~h}$ at room temperature. The membranes were incubated with primary antibodies against microtubule-associated protein 1 light chain 3B (LC3) (cat. no. ab192890), ATG7 (cat. no. ab52472) and $\beta$-actin (cat. no. ab8226) for $16 \mathrm{~h}$ at $4^{\circ} \mathrm{C}$ (all 1:1,000 and purchased from Abcam). Membranes were washed three times with TBST (0.01\% Tween) $(5 \mathrm{~min}$ each) and subsequently incubated with HRP-conjugated goat anti-rabbit IgG (1:5,000; Cell Signaling Technology, Inc.; cat. no. 7074) and anti-mouse $\operatorname{IgG}(1: 10,000$; Cell Signaling Technology, Inc.; cat. no. 7076S) secondary antibodies at room temperature for 1.5-2 h. Membranes were re-washed three times with TBST and the protein bands were visualized using an electrogenerated chemiluminescence detection system (Tanon-5200; Tanon Science and Technology Co., Ltd.). The rescue assays were performed by co-transfection with SNHG8 and mimics-miR-588.

Immunofluorescence staining. Cells were fixed with $4 \%$ paraformaldehyde (Sigma-Aldrich; Merck KGaA) for $20 \mathrm{~min}$ at $20^{\circ} \mathrm{C}$. Cells were subsequently permeabilized with $0.4 \%$ Triton X-100 for $5 \mathrm{~min}$ at room temperature, and blocked with $5 \% \mathrm{BSA}$ at $20^{\circ} \mathrm{C}$ for $30 \mathrm{~min}$. Cells were washed three times with PBS and incubated with LC3 primary antibody (1:100; Abcam; cat. no. ab192890) overnight at $4^{\circ} \mathrm{C}$. Cells were re-washed three times with PBS (5 min each) and subsequently incubated with AlexaFluor ${ }^{\circledR}$ 488-conjugated secondary antibodies (1:100; Abcam; cat. no. ab150077) for $1 \mathrm{~h}$ at $37^{\circ} \mathrm{C}$. Finally, cells were re-washed three times with PBS. Nuclei were stained with DAPI for $3 \mathrm{~min}$ at room temperature and cells were washed three times with PBS, prior to observation under a confocal microscope (magnification, $\mathrm{x} 400$; Olympus 
Corporation). Immunofluorescence analysis was performed using ImageJ v1.52 (National Institutes of Health).

Cell proliferation assay. Cell proliferation was assessed via the Cell Counting Kit-8 (CCK-8) assay (Beijing Solarbio Science \& Technology Co., Ltd.). HCT116 and SW480 cells were seeded into 96 -well plates at a density of $0.5-1 \times 10^{4}$ cells/well. Following transfection, cells were cultured for 24,48 and $72 \mathrm{~h}$. Cells were subsequently incubated with $10 \mu \mathrm{l} \mathrm{CCK}-8$ reagent for $2 \mathrm{~h}$ at $37^{\circ} \mathrm{C}$ and cell proliferation was analyzed at a wavelength of $450 \mathrm{~nm}$.

Dual-luciferase reporter assay. The binding sites were constructed using the StarBase database (http://starbase. sysu.edu.cn/). The luciferase reporter vector pmirGLO was purchased from Promega Corporation. Wild-type (WT) ATG7 (ATG7-WT) and the mutant (MUT) sequence of the miR-588 binding site of the 3'-UTR of ATG7 (ATG7-MUT) were cloned and inserted into the luciferase gene sequence. The WT SNHG8 luciferase reporter construct (SNHG8-WT) and mutant site (SNHG8-MUT) were constructed by Shanghai GenePharma Co., Ltd. The following sequences were used: ATG7-WT, 5'-CUGCUGCCCAGGAGUGGCCAG-3' and ATG7-MUT, 5'-CUGGAGCGGAGGAGCACCGGUG-3'; and SNHG8-WT, 5'-CGUCUGUGUCAUGUGGCCAU-3'; and SNHG8-MUT, 5'-CGUCUGUGUCAACACCGGUU-3'. Luciferase activity was measured $24 \mathrm{~h}$ after transfection and was detected using the Luciferase Assay System kit (Promega Corporation), according to the manufacturer's protocol. Firefly luciferase activity was normalized to Renilla luciferase activity.

Pull-down assay. Cellular lysates $(50 \mu \mathrm{l})$ were pulled down using biotinylated control (NC-Bio), miR-588 (miR-588-Bio) or miR-588 probes containing mutations in the SNHG8-binding site (miR-588-Bio-MUT). CRC cells (HCT116 and SW480) were cultivated with miR-588-Bio or miR-588-Bio-MUT (Thermo Fisher Scientific, Inc.). Cells were lysed in lysis buffer (Thermo Fisher Scientific, Inc.) and the lysate was subsequently cultivated with magnetic beads (50 $\mu \mathrm{l}$; Thermo Fisher Scientific, Inc.; cat. no. 65305) and RNA probe. The immunoprecipitate was obtained via magnetic forces and centrifugation at $1,000 \mathrm{x} \mathrm{g}$ for $20 \mathrm{~min}$ at room temperature, and was washed using the Pierce ${ }^{\mathrm{TM}}$ Magnetic RNA Pull-Down kit (Thermo Fisher Scientific, Inc.; cat. no. 20164). RNA was prepared using TRIzol ${ }^{\circledR}$ reagent, and SNHG8 enrichment was measured via PCR analysis. PCR was performed as aforementioned (RT-qPCR). The following probes were used: miR-588-Bio, 3'-CAAGAUUGGGUAACACCGGUU-5' and miR-588-Bio-MUT, 3'-CAUCUUUGGGUAUGUGGC CAU-5'.

Fluorescence in situ hybridization (FISH). Visualization of lncRNA localization in HCT116 and SW480 cells was assessed via FISH. HCT116 and SW480 cells were seeded into 6-wells plates and cultured until they reached $70 \%$ confluence. Cells were washed three times with PBS and subsequently fixed with $4 \%$ paraformaldehyde for $30 \mathrm{~min}$ at room temperature. FISH was performed using the BersinBio ${ }^{\mathrm{TM}}$ RNA FISH kit (BersinBio; cat. no. Bes-nRRF-HSA000002) according to the manufacturer's protocol. The RNase inhibitor was purchased from Thermo Fisher Scientific, Inc. (cat. no. AM2694). T7 RNA polymerase was purchased from Xinhai Gene Testing Co., Ltd. (cat. no. A3601A). Restriction enzyme (T7) was used to linearize plasmid. RT-qPCR was performed as aforementioned. The SNHG8 FISH probe (length, 2,067 nt) (DIG-dUTP and DIG RNA Labeling kit; Wolcavi Biotech; cat. no. 11175025910) was constructed. Cells were maintained in prehybridization solution (BersinBio) and hybridized with RNA FISH solution (Wuhan Servicebio Technology Co., Ltd.), containing SNHG8 FISH probe (3'-AATTCAAAT GTTCGTACG-5'). Subsequently, cells were stained with 6-diamidino-2-phenylindole dye solution for $5 \mathrm{~min}$ at room temperature ( $5 \mu \mathrm{g} / \mathrm{ml}$; Beyotime Institute of Biotechnology) and observed under a fluorescence microscope (magnification, x400; Olympus Corporation).

Bioinformatics analysis. The Cancer Genome Atlas (TCGA) dataset [colon adenocarcinoma (COAD)] was obtained and analyzed using the bioinformatics website UALCAN (http://ualcan.path.uab.edu/), including 41 normal tissues and 286 primary CRC tumor tissues. TargetScan (http://www. targetscan.org/mamm_31/) and StarBase (http://www.sysu. edu.cn/) were used to predict potential binding sites.

Statistical analysis. Statistical analysis was performed using SPSS 21.0 software (IBM Corp.). All experiments were performed in triplicate and data are presented as the mean \pm standard deviation. Unpaired Student's t-test was used to compare differences between two groups, while one-way ANOVA and Bonferroni post hoc test were used to compare differences between multiple groups. $\mathrm{P}<0.05$ was considered to indicate a statistically significant difference.

\section{Results}

SNHG8 expression is upregulated in CRC tissues and cell lines. To further investigate SNHG8 expression in CRC, TCGA database was analyzed (41 normal tissues and 286 primary CRC tumor tissues). The results demonstrated that SNHG8 expression was significantly upregulated in CRC tissues compared with in normal tissues $(\mathrm{P}<0.001$; Fig. 1A), indicating that abnormal SNHG8 expression may serve an important role in patients with CRC. RT-qPCR analysis was performed to detect SNHG8 expression in colorectal cell lines (HCT116, FHC, HCT8, HT29 and SW480). The results demonstrated that SNHG8 expression was significantly upregulated in CRC cells compared with FHC cells $(\mathrm{P}<0.05$; Fig. 1B). In addition, the subcellular distribution of SNHG8 in CRC cells was assessed. The results indicated that SNHG8 was predominantly located in the cytoplasm of HCT116 and SW480 cells (Fig. 2A and B).

SNHG8 promotes the proliferation of CRC cells. To determine the effect of SNHG8 on the proliferation of CRC cells, SNHG8 overexpressing cell lines were constructed using transfecting plasmids, and the transfection increased efficiency was 35-fold and 42-fold in HCT116 and SW480 cells, respectively $(\mathrm{P}<0.01$; Fig. $3 \mathrm{~A}$ and $\mathrm{B})$. The CCK-8 assay was performed to assess the effect of SNHG8 on the proliferation of CRC cells. The result demonstrated that overexpression of 
A

SNHG8 expression in COAD based on sample type

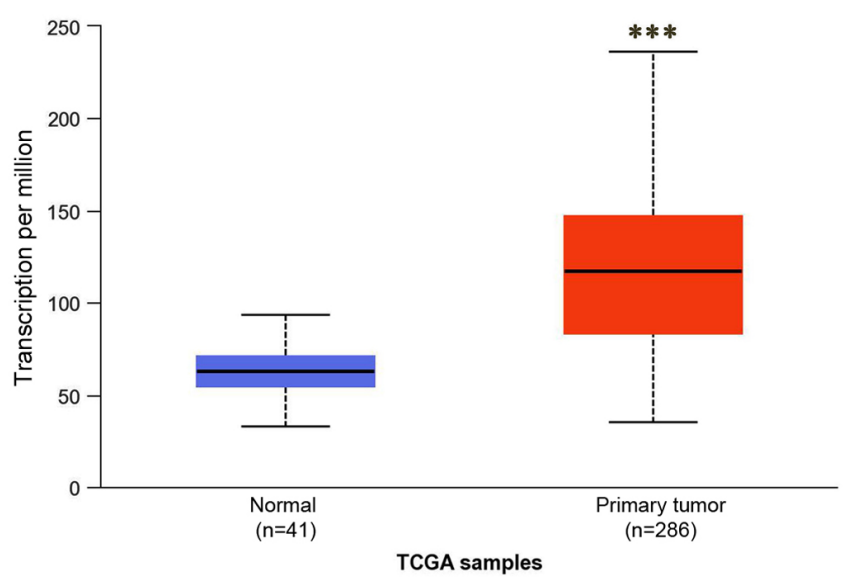

B

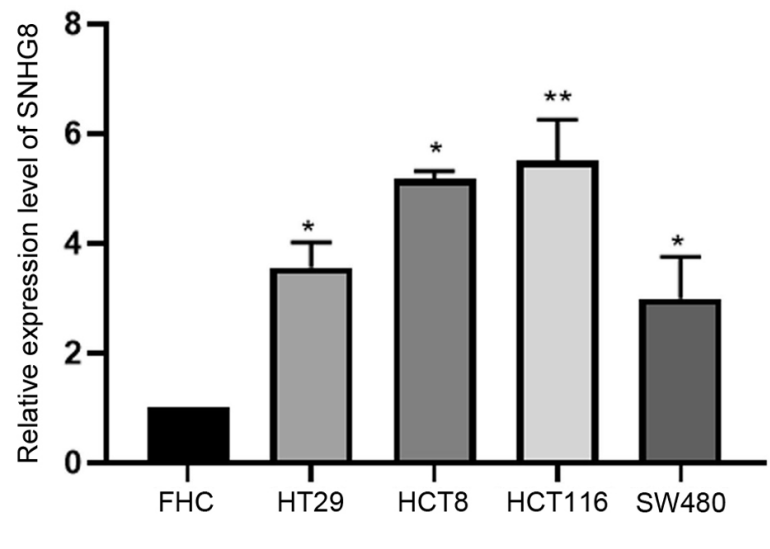

Figure 1. SNHG8 expression in COAD based on sample type. (A) lncRNA SNHG8 expression was significantly upregulated in primary CRC tumor tissues compared with normal tissues in The Cancer Genome Atlas COAD dataset (41 normal tissues and 286 primary CRC tumor tissues). (B) Reverse transcription-quantitative PCR analysis was performed to detect SNHG8 expression in CRC cell lines, HT29, HCT8, HCT116 and SW480, and FHC cells were used as the control group. Data are presented as the mean \pm standard deviation $(\mathrm{n}=3) .{ }^{*} \mathrm{P}<0.05,{ }^{* *} \mathrm{P}<0.01,{ }^{* * * *} \mathrm{P}<0.001$ vs. control. IncRNA, long non-coding RNA; SNHG8, small nucleolar RNA host gene 8; CRC, colorectal cancer; COAD, colon adenocarcinoma.

A

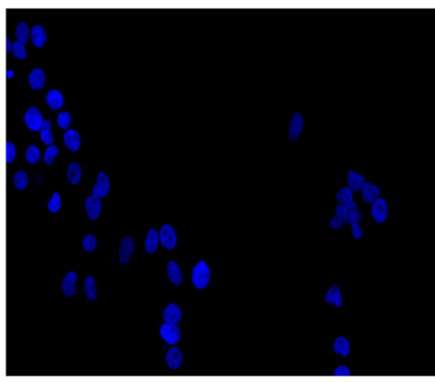

DAPI

B

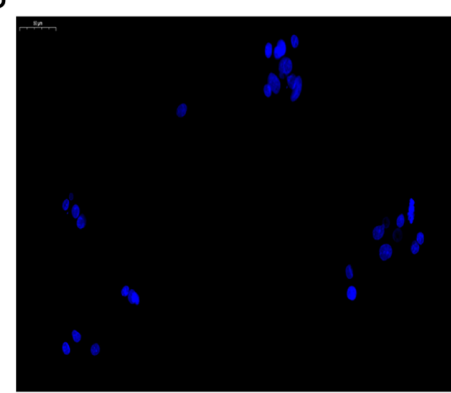

DAPI

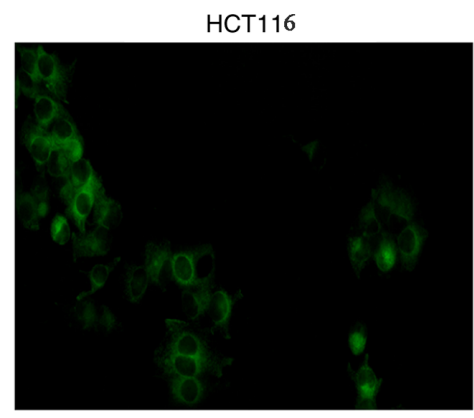

SNHG8

SW480

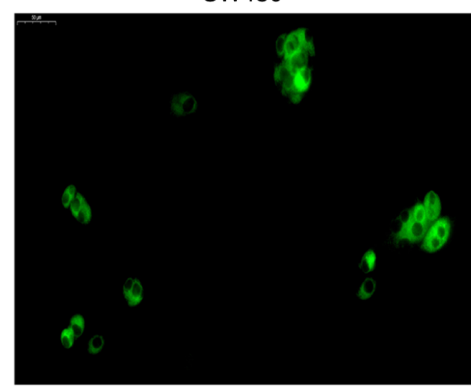

SNHG8

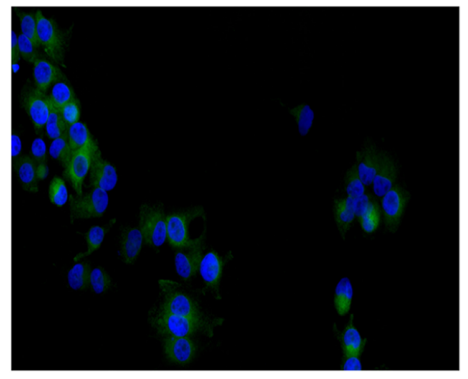

Merged

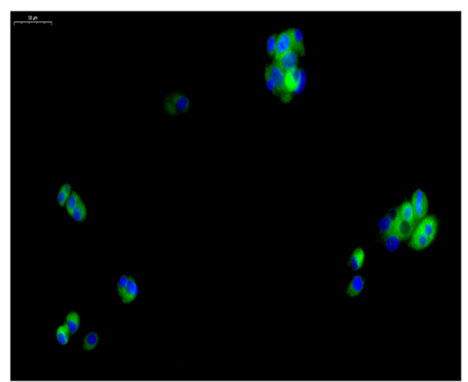

Merged

Figure 2. SNHG8 is mainly localized in the cytoplasm. SNHG8 was predominantly localized in the cytoplasm of colorectal cancer (A) HCT116 and (B) SW480 cells (magnification, $\mathrm{x} 400)$. Data are presented as the mean \pm standard deviation $(\mathrm{n}=3)$. SNHG8, small nucleolar RNA host gene 8 .

SNHG8 increased the proliferation of HCT116 and SW480 cells $(\mathrm{P}<0.05$; Fig. $3 \mathrm{C}$ and $\mathrm{D})$.

SNHG8 promotes autophagy in CRC cells. Increasing evidence suggest that autophagy plays a vital role in tumor progression (23). To investigate the association between SNHG8 and autophagy, ATGs were detected in the CRC cell lines. The results demonstrated that overexpression of SNHG8 enhanced the conversion of LC3-I to LC3-II in HCT116 and SW480 cells and quantitative analysis was performed, respectively $(\mathrm{P}<0.05$; Fig. 4A and B). In addition, immunofluorescence analysis demonstrated that overexpression of SNHG8 increased LC3 puncta in both HCT116 and SW480 cells ( $\mathrm{P}<0.01$; Fig. 4C). Taken together, these results suggest that SNHG8 promotes autophagy in CRC cells.

SNHG8 regulates autophagy by upregulating ATG7 expression in CRC cells. Previous studies have demonstrated that autophagy 

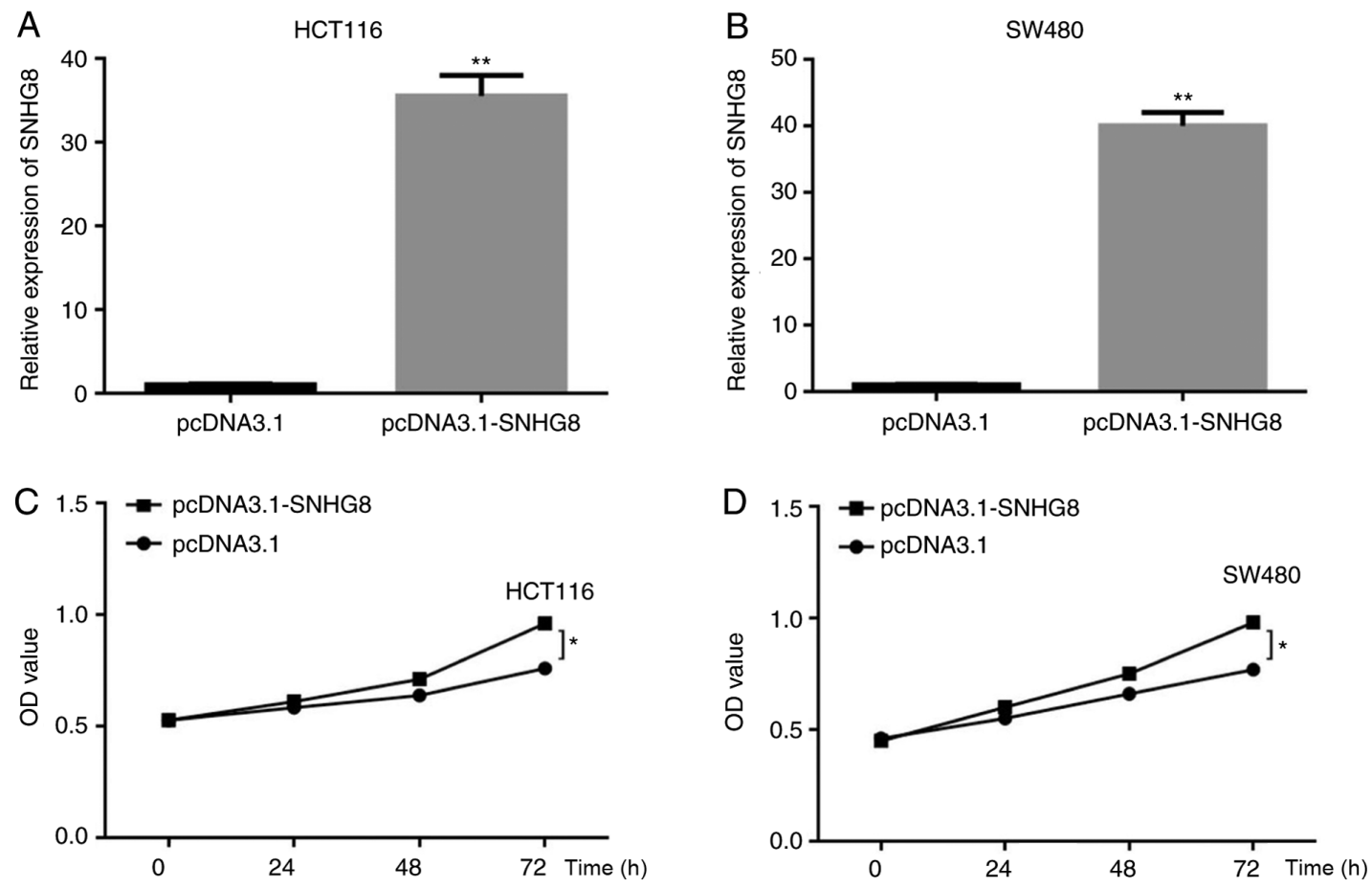

Figure 3. SNHG8 promotes the proliferation of colorectal cancer cells. Transfection of SNHG8 resulted in changes of 35-fold and 42-fold compared with the negative control (pcDNA3.1) in (A) HCT116 and (B) SW480 cells. Cell Counting Kit-8 assay demonstrated that overexpression of SNHG8 increased the proliferation of (C) HCT116 and (D) SW480 cells. Data are presented as the mean \pm standard deviation ( $\mathrm{n}=3) .{ }^{*} \mathrm{P}<0.05,{ }^{* * *} \mathrm{P}<0.01$ vs. control. SNHG8, small nucleolar RNA host gene 8; OD, optical density.

is a dynamic and continuous process that involves ATGs $(24,25)$, with several ATGs participating in autophagosome formation (23). To further investigate the molecular mechanisms, RT-qPCR analysis was performed to detect the potential targets, including ATG3, ATG5, ATG7, ATG10 and ATG12. The results demonstrated that overexpression of SNHG8 significantly upregulated ATG7 expression in HCT116 and SW480 cells $(\mathrm{P}<0.01$; Fig. 5A). Consistent with these results, ATG7 protein expression was markedly upregulated following overexpression of SNHG8 (Fig. 5B and C). Subsequently, si-ATG7 cell lines were constructed, and RT-qPCR and western blot analyses were performed to detect ATG7 mRNA and protein expression levels following transfection with si-ATG7 or si-NC, respectively (HCT116, P<0.001; SW480, P<0.01; Fig. 5D and E). Specific siRNA for ATG7 was used to perform the rescue experiments, and quantitative analysis was performed ( $\mathrm{P}<0.05$; Fig. $5 \mathrm{~F}$ and $\mathrm{G})$. Collectively, these results suggest that SNHG8 promotes autophagy in CRC cells by upregulating ATG7 expression.

miR-588 inhibits ATG7 expression. 1ncRNA SNHG8 has been reported to regulate gene expression as a ceRNA by sponging specific miRNAs (26). To further investigate the molecular mechanisms underlying SNHG8 in autophagy, the potential binding sites between ATG7 and miRNAs were analyzed. The TargetScan and StarBase databases demonstrated that miR-588 may have a potential binding site with SNHG8 or the 3'-UTR of ATG7 mRNA (Fig. 6A). The present study aimed to investigate whether miR-588 can inhibit ATG7 expression, and thus miR-588 overexpressing cell lines were constructed using miR-588 mimics. The transfection efficiency resulted in 6.3-fold and 5.9-fold increases in HCT116 and SW480 cells, respectively (P<0.01; Fig. 6B). The results demonstrated that miR-588 significantly inhibited ATG7 expression (Fig. 6C and D). The dual-luciferase reporter assay was performed to confirm the binding sites between miR-588 and ATG7 (P<0.05; Fig. 6E and F).

SNHG8 mediates ATG7 expression via miR-588. To further verify the underlying molecular mechanism of SNHG8 in CRC cells, the present study investigated the molecular mechanisms of SNHG8 in autophagy and analyzed the potential binding sites between SNHG8 and miR-588 using the StarBase database (Fig. 7A). RT-qPCR analysis was performed to detect miR-588 expression following overexpression of SNHG8. The results demonstrated that miR-588 expression was inhibited following overexpression of SNHG8 $(\mathrm{P}<0.01$; Fig. 7B). The dual-luciferase reporter assay was performed to confirm the association between miR-588 and SNHG8 $(\mathrm{P}<0.05$; Fig. $7 \mathrm{C}$ and $\mathrm{D})$. In addition, the pull-down assay was performed to assess the effect of miR-588 on SNHG8. The results demonstrated that miR-588 can bind with SNHG8 $(\mathrm{P}<0.05$; Fig. 7E and F). Western blot analysis was performed to detect ATG7 protein expression in cells under different conditions (Fig. 7G and $\mathrm{H}$ ). The results demonstrated that overexpression of miR-588 inhibited upregulation of ATG7 expression via SNHG8. Taken together, these results suggest that SNHG8 regulates the occurrence of autophagy through the regulation of ATG7 via miR-588.

\section{Discussion}

It is estimated that 135,430 new cases of CRC are diagnosed and 50,260 CRC-associated mortalities are reported per year 
A
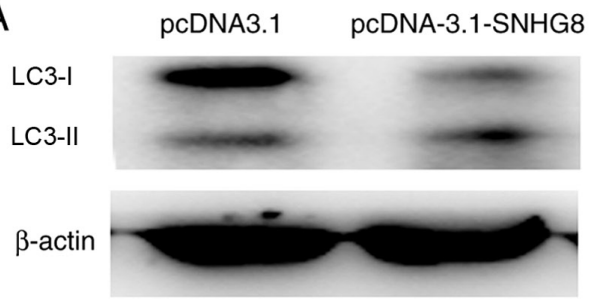

HCT-116

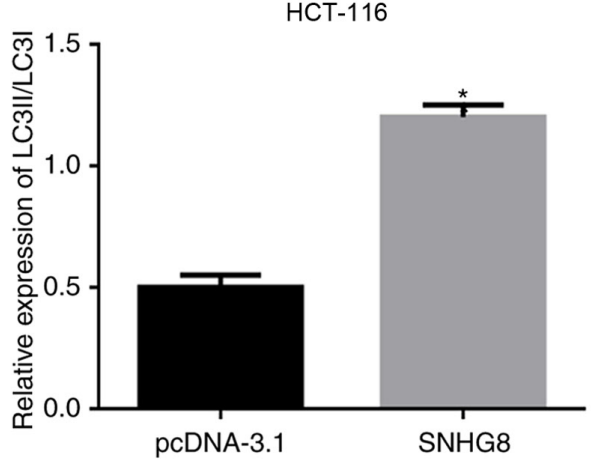

B LC3-I

LC3-II

$\beta$-actin
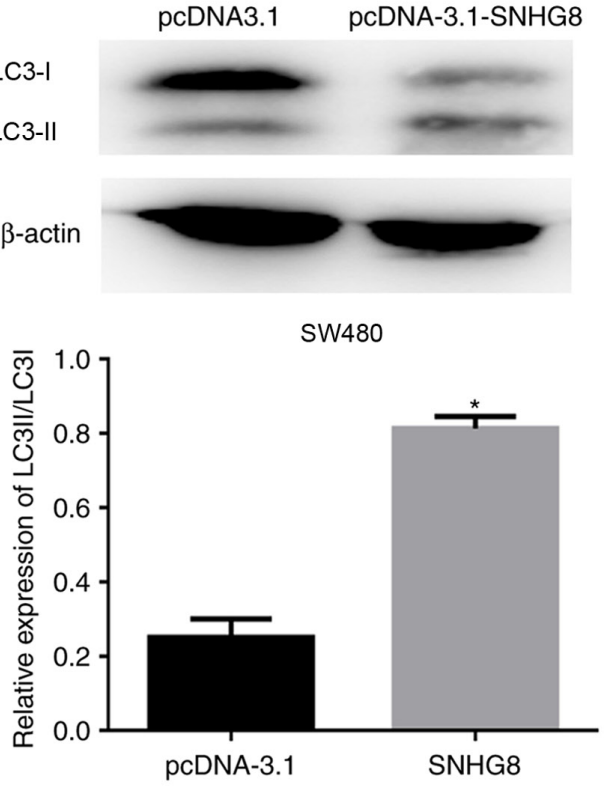

C
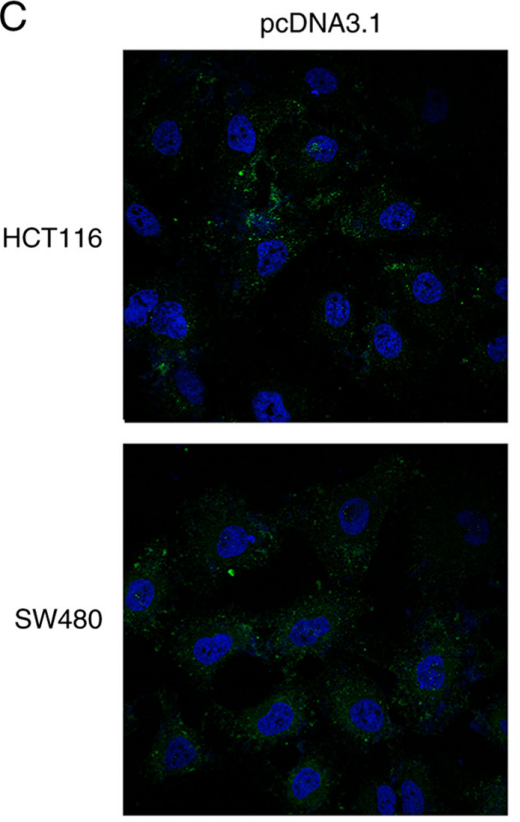

pcDNA3.1-SNHG8
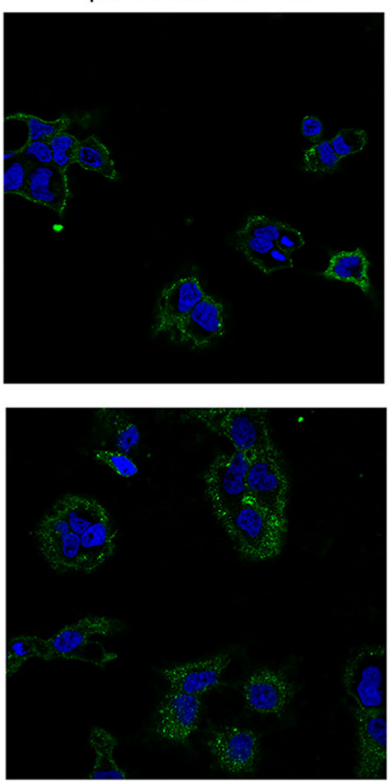
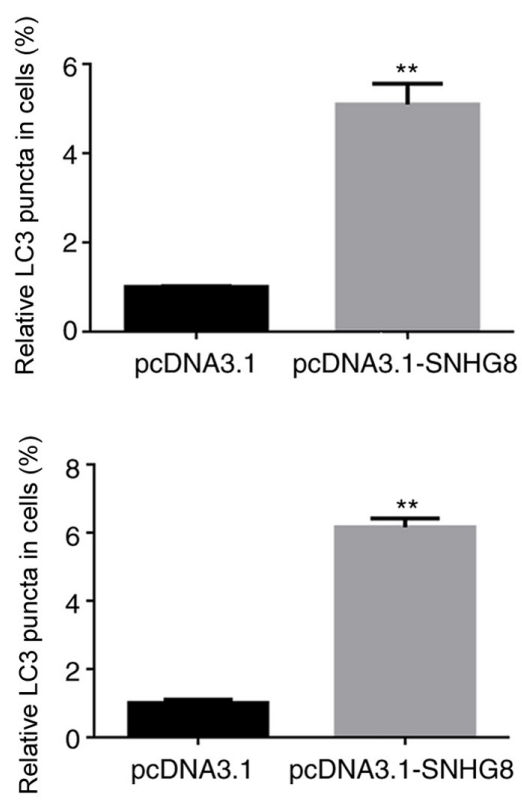

Figure 4. SNHG8 promotes autophagy in colorectal cancer cells. Western blot analysis demonstrated that overexpression of SNHG8 increased the conversion of LC3-I to LC3-II in both (A) HCT116 and (B) SW480 cells. (C) Immunofluorescence analysis demonstrated that overexpression of SNHG8 increased LC3 puncta in both HCT116 and SW480 cells, and statistical comparison was performed using ImageJ software. Data are presented as the mean \pm standard deviation $(\mathrm{n}=3) .{ }^{*} \mathrm{P}<0.05,{ }^{* *} \mathrm{P}<0.01$ vs. control. SNHG8, small nucleolar RNA host gene 8; LC3, microtubule-associated protein 1 light chain 3B.

in the United States $(1,2)$. Despite recent advancements in the treatment methods for CRC, the prognosis for patients with CRC remains unsatisfactory (27). Thus, the identification of novel biomarkers and therapeutic targets for CRC are urgently required.

Autophagy is a self-digestion process that eliminates harmful cargo to protect cells from stress. IncRNAs are a class of RNA molecules that play important roles in tumor progression (28). Increasing evidence suggest that lncRNAs are involved in the tumor cell biology $(29,30)$. SNHG8 is a novel oncogene that acts as a target to prevent tumor progression, and some studies have indicated that SNHG8 induces cell proliferation, invasion and metastasis in different types of tumors $(13,14,31)$. Thus, the present study aimed to investigate the molecular mechanism of SNHG8 in CRC to provide a target for future clinical treatment.

The present study analyzed SNHG8 expression in CRC tissues using TCGA dataset. The results demonstrated that SNHG8 expression was abnormally expressed in CRC tissues compared with normal tissues, suggesting the involvement of SNHG8 in tumor progression. Previous studies had demonstrated that SNHG8 serves as an oncogene, whereby its expression is upregulated in several types of solid tumors, such as breast cancer and osteosarcoma $(13,14,31)$. In the present study, immunofluorescence and western blot analyses were performed to determine the molecular mechanisms of SNHG8 


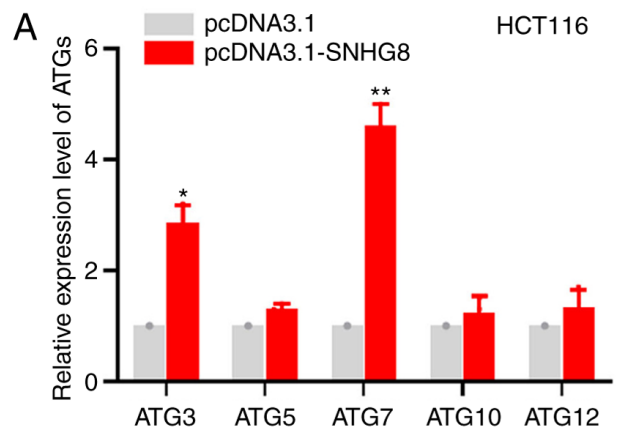

B
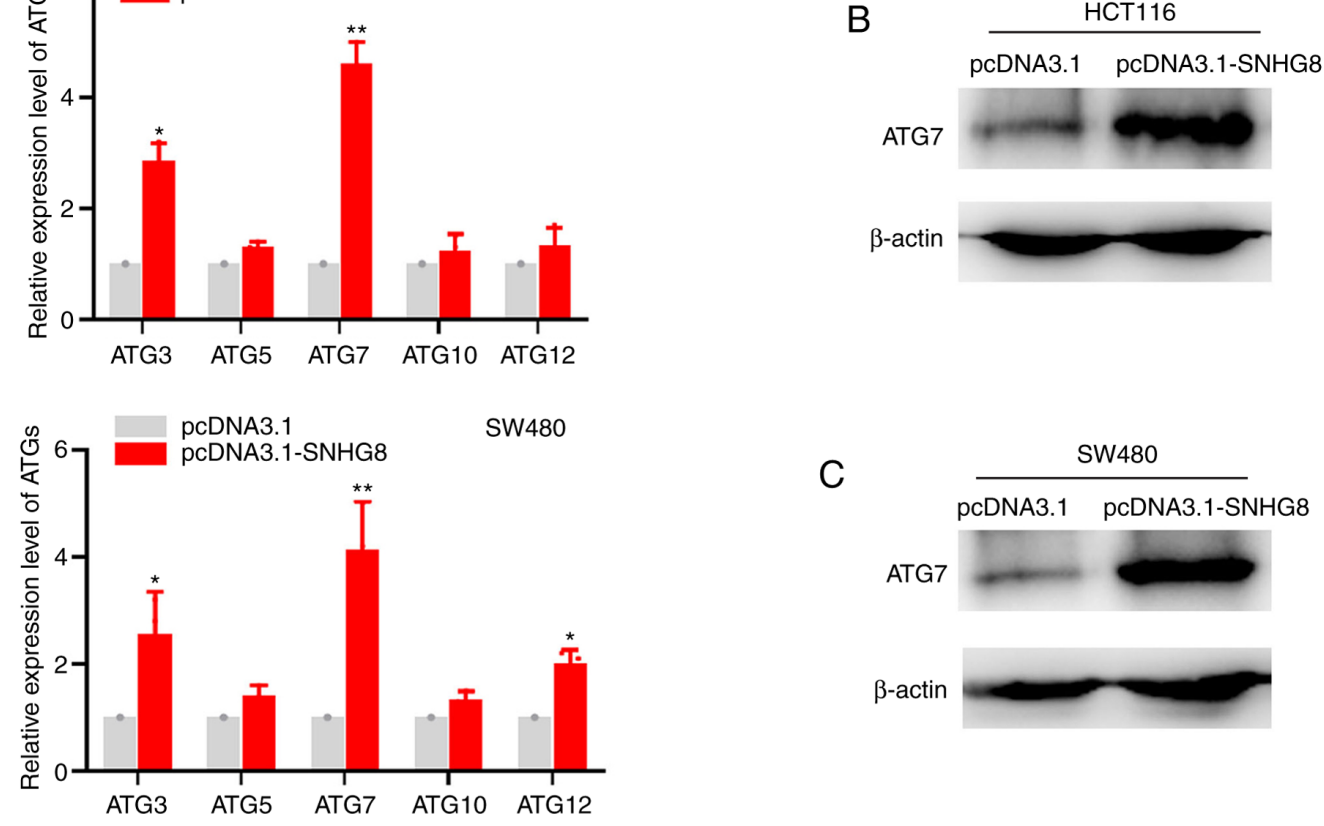

C
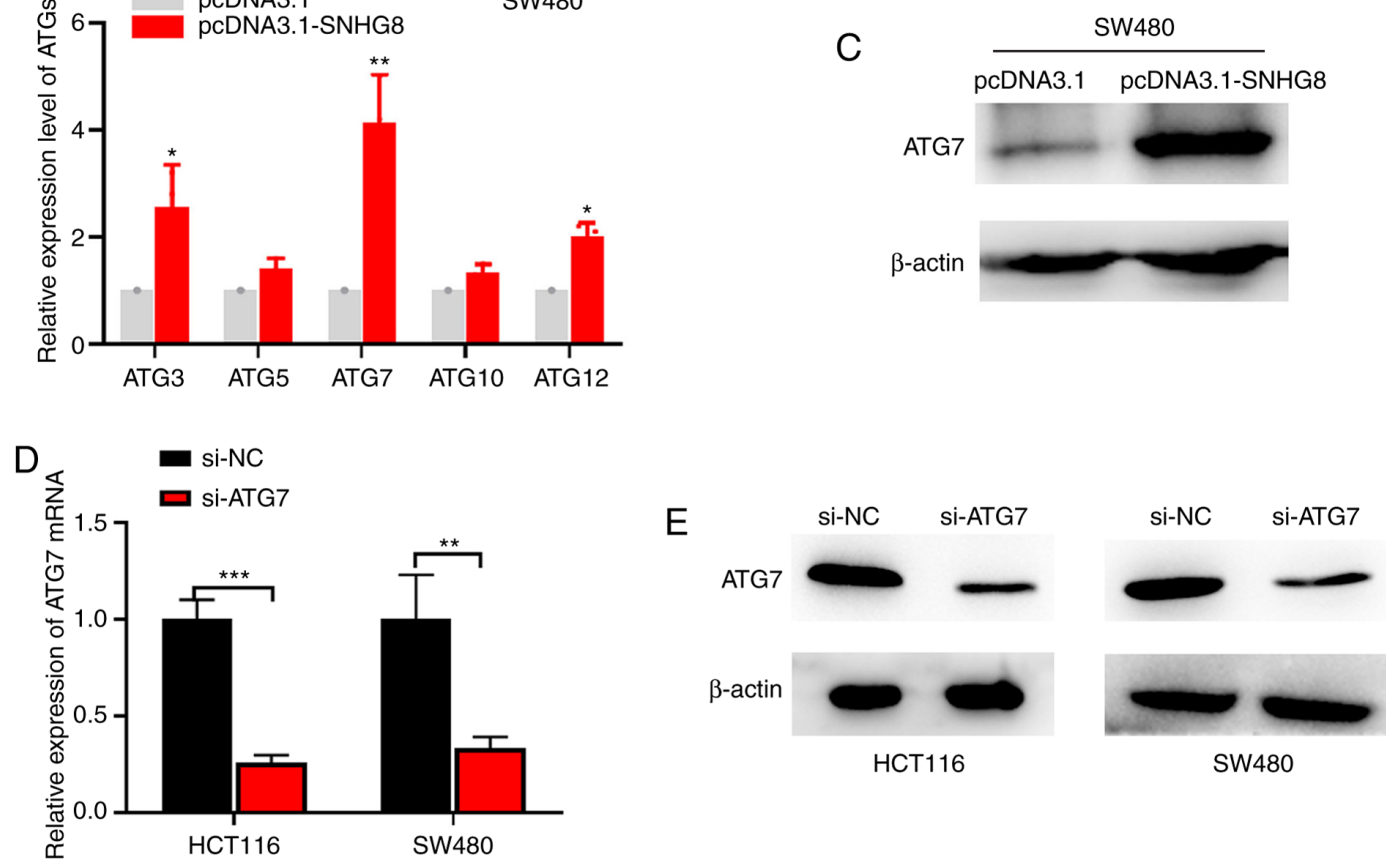

F

HCT116

G
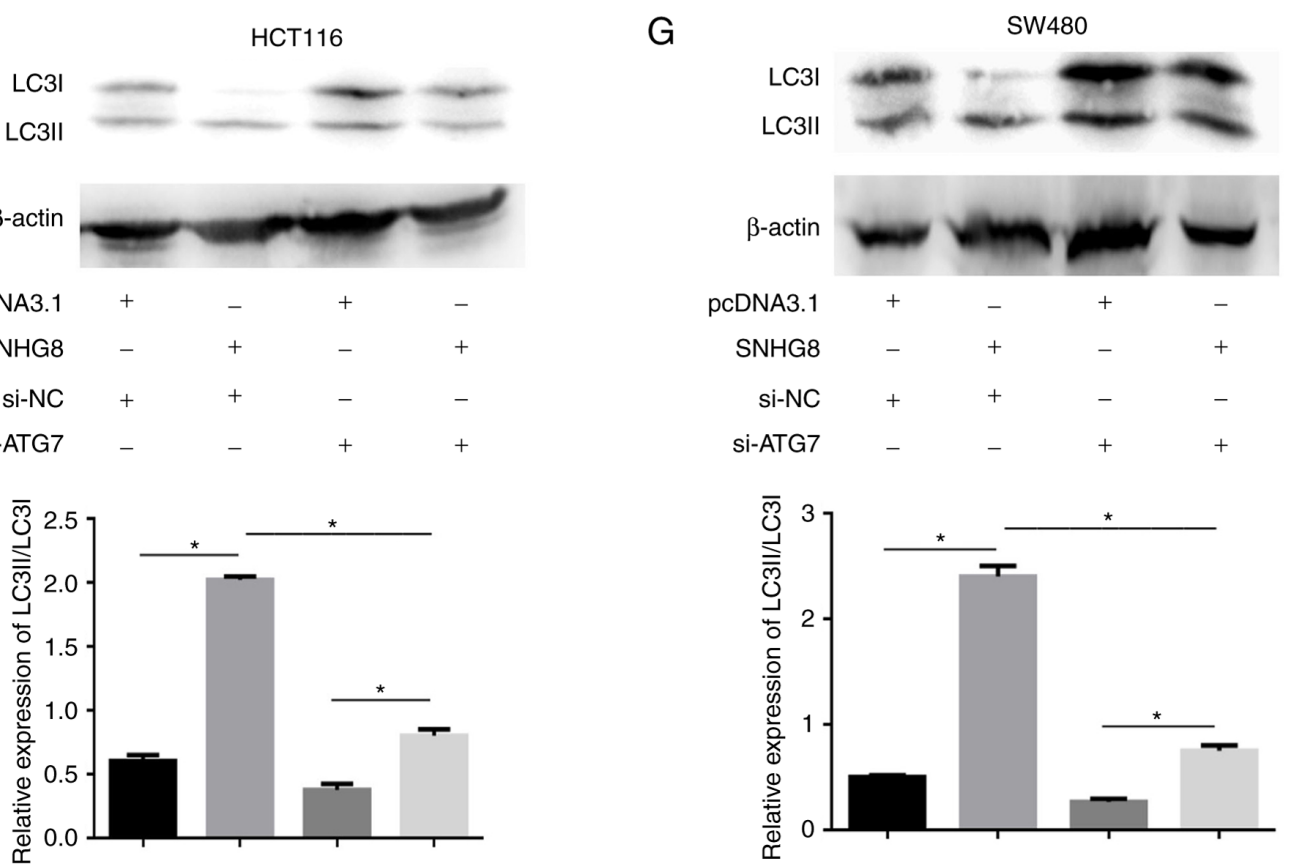

Figure 5. SNHG8 regulates autophagy by upregulating ATG7 expression in CRC cells. (A) RT-qPCR analysis was performed to detect potential regulatory targets, including ATG3, ATG5, ATG7, ATG10 and ATG12. Western blot analysis was performed to detect ATG7 protein expression following overexpression of SNHG8 in (B) HCT116 and (C) SW480 cells. (D) RT-qPCR and (E) western blot analyses were performed to detect ATG7 mRNA and protein expression levels following transfection with si-NC and si-ATG7, respectively. Rescue experiments demonstrated that SNHG8 promoted autophagy by upregulating

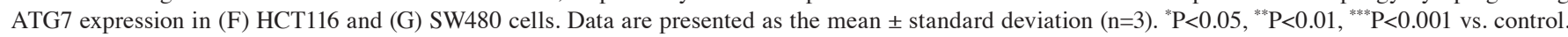
SNHG8, small nucleolar RNA host gene 8; ATG, autophagy-related gene; CRC, colorectal cancer; RT-qPCR, reverse transcription-quantitative PCR; si, small interfering; NC, negative control; LC3, microtubule-associated protein 1 light chain 3B. 


\section{A}

Target: $\quad$ 5' cugCUGCCCAGGAGUGGCCAg 3'

$$
\text { II: ||: }||||||
$$

miRNA: $\quad 3$ ' caaGAUUGGGUAACACCGGUu $\quad 5^{\prime}$

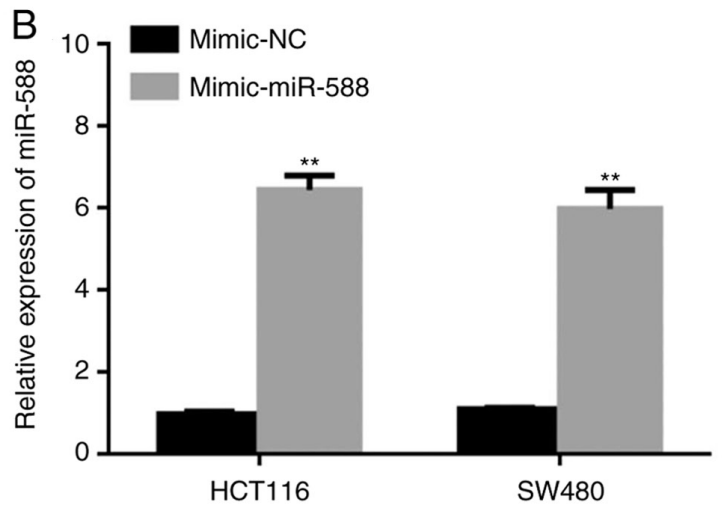

$\mathrm{D}$

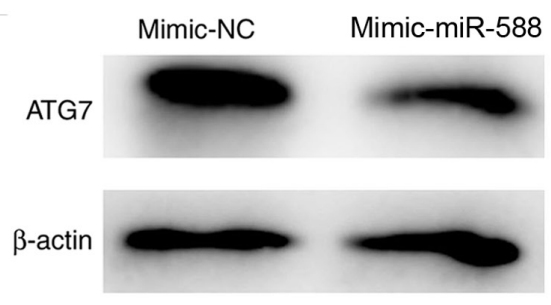

F

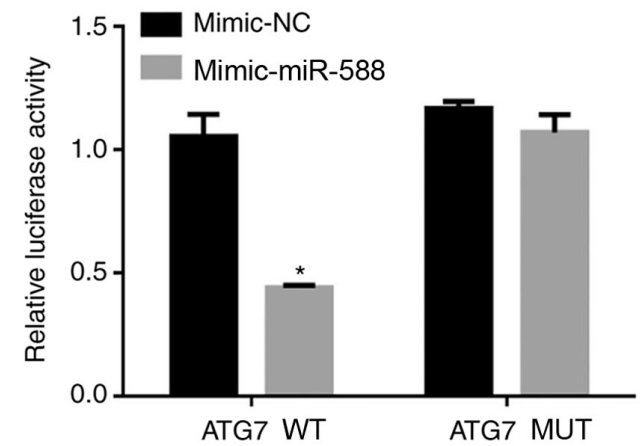

Figure 6. miR-588 inhibits ATG7 expression. (A) The StarBase database revealed that miR-588 has a potential binding site with the 3'-untranslated region of ATG7 mRNA. (B) The transfection efficiency resulted in 6.3-fold and 5.9-fold increases in HCT116 and SW480 cells. Western blot analysis demonstrated that miR-588 significantly inhibited ATG7 expression in (C) HCT116 and (D) SW480 cells. The dual-luciferase reporter assay confirmed the binding sites between miR-588 and ATG7 in (E) HCT116 and (F) SW480 cells. Mutant sites were used according to the StarBase database. Data are presented as the mean \pm standard deviation (n=3). ${ }^{*} \mathrm{P}<0.05,{ }^{* *} \mathrm{P}<0.01$ vs. mimic-NC. miR, microRNA; ATG, autophagy-related gene; NC, negative control; WT, wild-type; MUT, mutant.

in CRC cells. The results demonstrated that SNHG8 promoted CRC cell proliferation and autophagy. Recently, it has been demonstrated that autophagy is a protective process in cells, particularly under stress; however, the excessive activation of autophagy may result in cell death (13). Autophagy is a highly conserved process that exerts a protective mechanism to promote drug resistance of cells (32). Furthermore, autophagy activation can promote malignant migratory and invasive abilities $(33,34)$. However, the role of SNHG8 on CRC cellular autophagy remains unclear. Thus, the present study aimed to investigate the role of SNHG8 in autophagy.

In the present study, western blot and immunofluorescence analyses demonstrated that the level of autophagy was enhanced following overexpression of SNHG8. Autophagy is a complex process involving several ATGs, such as ATG3, ATG5, ATG7, ATG10 and ATG12, which are associated with tumor progression (35). A previous study demonstrated that IncRNAs can mediate ATG expression to promote autophagy in tumors (36). For example, IncRNA KCNQ1OT1 promotes autophagy via ATG3 in lung cancer (37). In addition, lncRNA MEG3 promotes autophagy via the miR-21/PTEN axis in nasopharyngeal carcinoma cells (38). Taken together, these findings suggest that lncRNAs play important roles in tumor-related autophagy.

To further investigate the molecular mechanisms of SNHG8 in autophagy, the expression levels of ATGs were measured, and ATG7 expression was upregulated in CRC cells following transfection with the SNHG8 plasmid. Taken together, the results of the present study demonstrated that ATG7 can be regulated by SNHG8.

LncRNAs sponge miRNAs to increase mRNA expression (39). miR-588 is an important miRNA that has been reported to regulate different types of tumors, such as breast cancer, and it a well-known prognostic marker $(18,19)$. However, the association between SNHG8 and miR-588 remains unclear. To further investigate the potential molecular mechanisms, the binding sites between SNHG8 and miR-588 were predicted using the StarBase database. Bioinformatics analysis revealed that SNHG8 is a target of miR-588, and miR-588 can target ATG7 mRNA. The dual-luciferase reporter assay was performed to confirm the association between miR-588 and ATG7 mRNA. The results of the dual-luciferase 
A

Target: 5 ' cgUCUGUGUCA-UGUGGCCAu 3

III: : || $\||\|\mid\|$

miRNA: $\quad 3^{\prime}$ caAGAUUGGGUACACCGGUu 5'
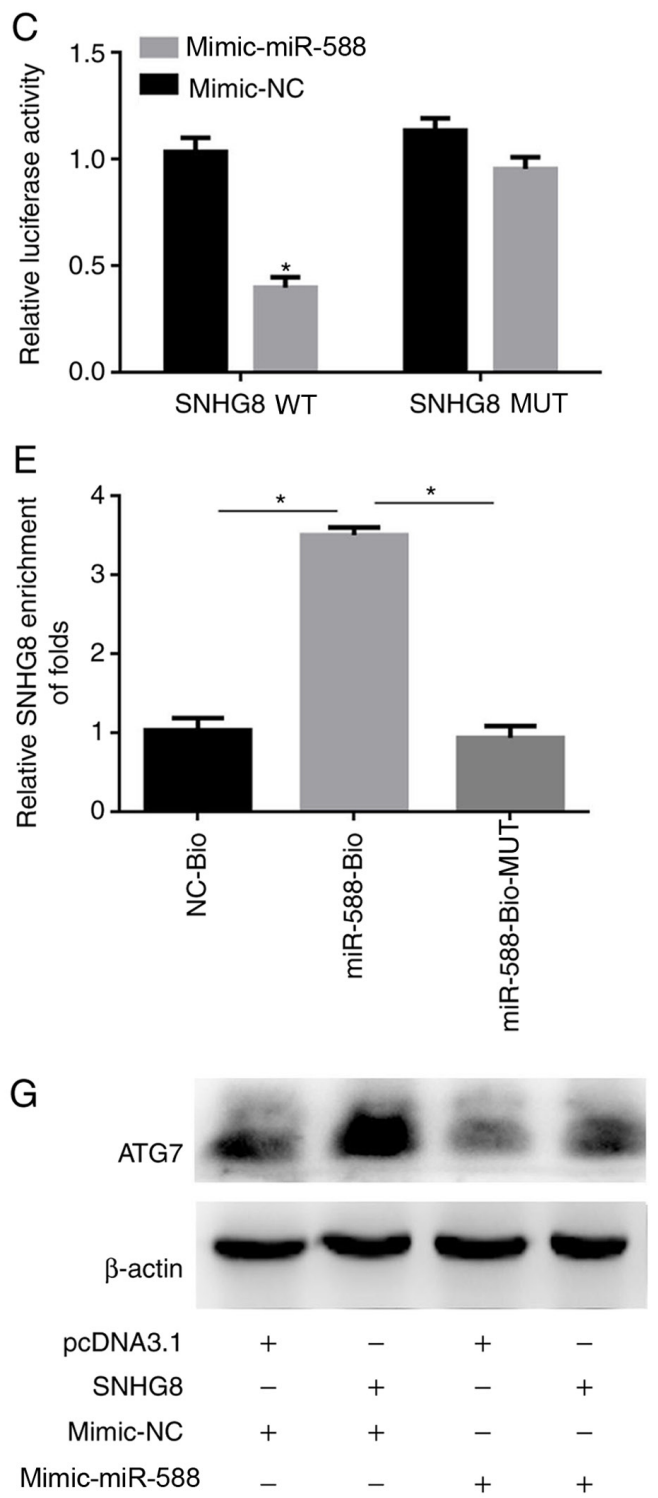

$B$
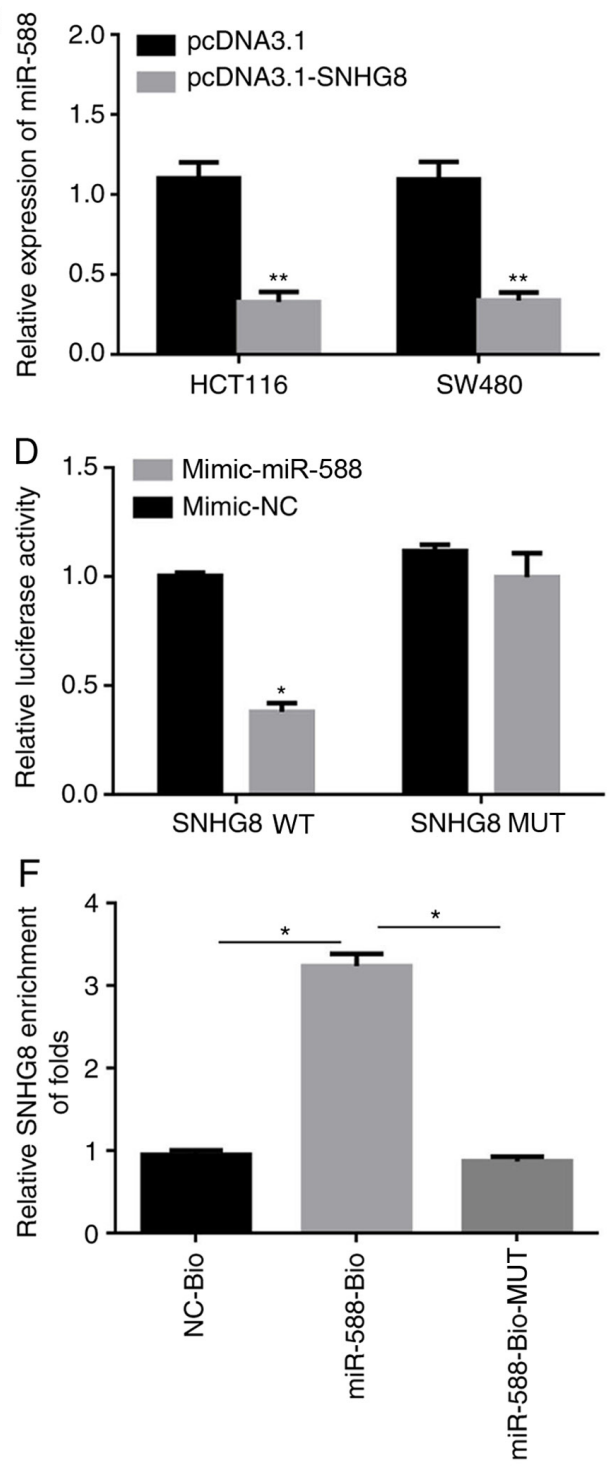

$\mathrm{H}$

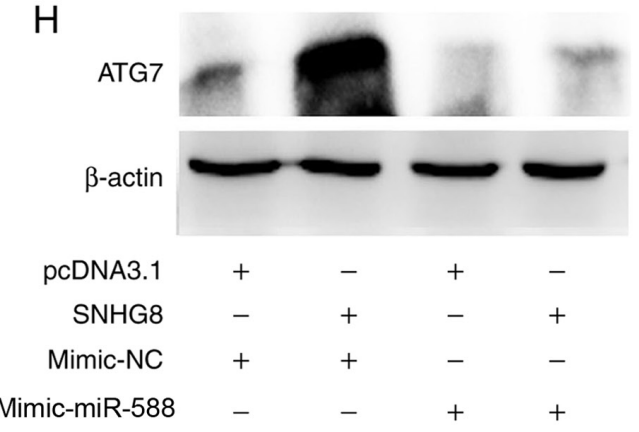

Figure 7. SNHG8 mediates ATG7 expression via miR-588. (A) The StarBase database revealed the potential binding sites between SNHG8 and miR-588. (B) Reverse transcription-quantitative PCR analysis demonstrated that miR-588 expression was inhibited following overexpression of SNHG8. The dual-luciferase reporter assay was performed to confirm the association between miR-588 and SNHG8 in (C) HCT116 and (D) SW480 cells. The pull-down assay was performed to confirm the association between miR-588 and SNHG8 in (E) HCT116 and (F) SW480 cells. Cellular lysates were pulled down using biotinylated control (NC-Bio), miR-588 (miR-588-Bio) or miR-588 probe containing mutations in the SNHG8-binding site (miR-588-Bio-MUT). Western blot analysis was performed to detect ATG7 protein expression in (G) HCT116 and (H) SW480 cells. Data are presented as the mean \pm standard deviation (n=3). "P<0.05, ${ }^{* *} \mathrm{P}<0.01$ vs. control. SNHG8, small nucleolar RNA host gene 8; ATG, autophagy-related gene; miR, microRNA; NC, negative control; WT, wild-type; MUT, mutant.

reporter and pull-down assays verified the association between SNHG8 and miR-588. Collectively, these results suggest that SNHG8 can target miR-588 and ATG7 is a target of miR-588. To further clarify the regulatory network, a rescue assay was performed, which proved that SNHG8 promotes autophagy via the miR-588/ATG7 axis. Taken together, the results of the present study suggest that SNHG8 can target miR-588 to inhibit its expression, which enhances ATG7 mRNA expression. 
The present study is not without limitations. For example, only two CRC cell lines were used to prove the generality of these results and investigate the molecular mechanisms in vivo. However, the results provided a novel target in the tumorigenesis of CRC.

In conclusion, the results of the present study demonstrated that SNHG8 expression was substantially upregulated in CRC cells and tissues. Furthermore, overexpression of SNHG8 enhanced autophagy in CRC cells via the miR-588/ATG7 axis. Notably, the present study identified a target of autophagy, which may provide a novel therapeutic target for CRC and a promising autophagy-related therapeutic method.

\section{Acknowledgements}

Not applicable.

\section{Funding}

No funding was received.

\section{Availability of data and materials}

All data generated or analyzed during this study are included in this published article.

\section{Authors' contributions}

$\mathrm{CH}, \mathrm{YF}$ and YC designed the present study. XL revised the manuscript for important intellectual content and provided final approval of the version to be published. $\mathrm{CH}$ performed the experiments and drafted the initial manuscript. XL and $\mathrm{CH}$ analyzed and interpreted the data, and confirm the authenticity of all the raw data. All authors have read and approved the final manuscript.

\section{Ethics approval and consent to participate}

Not applicable.

\section{Patient consent for publication}

Not applicable.

\section{Competing interests}

The authors declare that they have no competing interests.

\section{References}

1. Liu A and Liu L: Long non-coding RNA ZEB2-AS1 promotes proliferation and inhibits apoptosis of colon cancer cells via miR-143/bcl-2 axis. Am J Transl Res 11: 5240-5248, 2019.

2. Siegel RL, Miller KD and Jemal A: Cancer statistics, 2017. CA Cancer J Clin 67: 7-30, 2017.

3. Siegel RL, Miller KD, Fedewa SA, Ahnen DJ, Meester RGS, Barzi A and Jemal A: Colorectal cancer statistics, 2017. CA Cancer J Clin 67: 177-193, 2017.

4. Li X, Zhou Y, Li Y, Yang L, Ma Y, Peng X, Yang S, Liu J and Li H: Autophagy: A novel mechanism of chemoresistance in cancers. Biomed Pharmacother 119: 109415, 2019.
5. Li C, Lei B, Huang S, Zheng M, Liu Z, Li Z and Deng Y: H19 derived microRNA-675 regulates cell proliferation and migration through CDK6 in glioma. Am J Transl Res 7: 1747-1764, 2015.

6. Liu J, Tian W, Zhang W, Jia Y, Yang X, Wang Y and Zhang J: MicroRNA-142-3p/MALAT1 inhibits lung cancer progression through repressing $\beta$-catenin expression. Biomed Pharmacother 114: 108847, 2019.

7. Amaravadi R, Kimmelman AC and White E: Recent insights into the function of autophagy in cancer. Genes Dev 30: 1913-1930, 2016.

8. Luo M, Li Z, Wang W, Zeng Y, Liu Z and Qiu J: Long non-coding RNA H19 increases bladder cancer metastasis by associating with EZH2 and inhibiting E-cadherin expression. Cancer Lett 333: 213-221, 2013.

9. Ma H, Yuan L, Li W, Xu K and Yang L: The lncRNA H19/miR-193a-3p axis modifies the radio-resistance and chemotherapeutic tolerance of hepatocellular carcinoma cells by targeting PSEN1. J Cell Biochem 119: 8325-8335, 2018.

10. Tang B, Bao N, He G and Wang J: Long noncoding RNA HOTAIR regulates autophagy via the miR-20b-5p/ATG7 axis in hepatic ischemia/reperfusion injury. Gene 686: 56-62, 2019.

11. Si Y, Yang Z, Ge Q, Yu L, Yao M, Sun X, Ren Z and Ding C: Long non-coding RNA Malat1 activated autophagy, hence promoting cell proliferation and inhibiting apoptosis by sponging miR-101 in colorectal cancer. Cell Mol Biol Lett 24: $50,2019$.

12. Zhang P, Li S, Chen Z, Lu Y and Zhang H: lncRNA SNHG8 promotes proliferation and invasion of gastric cancer cells by targeting the miR-491/PDGFRA axis. Hum Cell 33: 123-130, 2020.

13. Fan D, Qiu B, Yang XJ, Tang HL, Peng SJ, Yang P, Dong YM, Yang L, Bao GQ and Zhao HD: IncRNA SNHG8 promotes cell migration and invasion in breast cancer cell through miR-634/ZBTB20 axis. Eur Rev Med Pharmacol Sci 24: 11639-11649, 2020.

14. Miao W, Lu T, Liu X, Yin W and Zhang H: lncRNA SNHG8 induces ovarian carcinoma cells cellular process and stemness through Wnt/ $\beta$-catenin pathway. Cancer Biomark 28: 459-471, 2020.

15. Ying SY, Chang DC and Lin SL: The microRNA (miRNA): Overview of the RNA genes that modulate gene function. Mol Biotechnol 38: 257-268, 2008.

16. Shukla GC, Singh J and Barik S: MicroRNAs: Processing, maturation, target recognition and regulatory functions. Mol Cell Pharmacol 3: 83-92, 2011.

17. Qian L, Lin L, Du Y, Hao X, Zhao Y and Liu X: MicroRNA-588 suppresses tumor cell migration and invasion by targeting GRN in lung squamous cell carcinoma. Mol Med Rep 14: 3021-3028, 2016.

18. Yu M, Zhang X, Li H, Zhang P and Dong W: MicroRNA-588 is downregulated and may have prognostic and functional roles in human breast cancer. Med Sci Monit 23: 5690-5696, 2017.

19. Kohlhapp FJ, Mitra AK, Lengyel E and Peter ME: MicroRNAs as mediators and communicators between cancer cells and the tumor microenvironment. Oncogene 34: 5857-5868, 2015.

20. Masters JR, Thomson JA, Daly-Burns B, Reid YA, Dirks WG, Packer P, Toji LH, Ohno T, Tanabe H, Arlett CF, et al: Short tandem repeat profiling provides an international reference standard for human cell lines. Proc Natl Acad Sci USA 98: 8012-8017, 2001.

21. Li HR, Shagisultanova EI, Yamashita K, Piao Z, Perucho M and Malkhosyan SR: Hypersensitivity of tumor cell lines with microsatellite instability to DNA double strand break producing chemotherapeutic agent bleomycin. Cancer Res 64: 4760-4767, 2004.

22. Livak KJ and Schmittgen TD: Analysis of relative gene expression data using real-time quantitative PCR and the 2(-Delta Delta C(T)) method. Methods 25: 402-408, 2001.

23. Mukhopadhyay S, Biancur DE, Parker SJ, Yamamoto K, Banh RS, Paulo JA, Mancias JD and Kimmelman AC: Autophagy is required for proper cysteine homeostasis in pancreatic cancer through regulation of SLC7A11. Proc Natl Acad Sci USA 118: e2021475118, 2021.

24. Xu JL, Yuan L, Tang YC, Xu ZY, Xu HD, Cheng XD and Qin JJ: The role of autophagy in gastric cancer chemoresistance: Friend or foe? Front Cell Dev Biol 8: 621428, 2020.

25. Yun CW, Jeon J, Go G, Lee JH and Lee SH: The dual role of autophagy in cancer development and a therapeutic strategy for cancer by targeting autophagy. Int J Mol Sci 22: 179, 2020. 
26. Shi Z, Zhang H, Jie S, Yang X, Huang Q, Mao Y and Zhang Y: Long non-coding RNA SNHG8 promotes prostate cancer progression through repressing miR-384 and up-regulating HOXB7. J Gene Med: e3309, 2021.

27. Yu X, Zhu L, Liu J, Xie M, Chen J and Li J: Emerging role of immunotherapy for colorectal cancer with liver metastasis. Onco Targets Ther 13: 11645-11658, 2020.

28. Jin KT, Tao XH, Fan YB and Wang SB: Crosstalk between oncolytic viruses and autophagy in cancer therapy. Biomed Pharmacother 134: 110932, 2021.

29. Yan X, Hu Z, Feng Y, Hu X, Yuan J, Zhao SD, Zhang Y, Yang L, Shan W, He Q, et al: Comprehensive genomic characterization of long Non-coding RNAs across human cancers. Cancer Cell 28 : 529-540, 2015

30. Li CF, Li YC, Wang Y and Sun LB: The effect of lncRNA H19/miR-194-5p axis on the epithelial-mesenchymal transition of colorectal adenocarcinoma. Cell Physiol Biochem 50: 196-213, 2018.

31. Qu X, Li Y, Wang L, Yuan N, Ma M and Chen Y: IncRNA SNHG8 accelerates proliferation and inhibits apoptosis in HPV-induced cervical cancer through recruiting EZH 2 to epigenetically silence RECK expression. J Cell Biochem 121: 4120-4129, 2020.

32. Dikic I and Elazar Z: Mechanism and medical implications of mammalian autophagy. Nat Rev Mol Cell Biol 19: 349-364, 2018

33. Kroemer G: Autophagy: A druggable process that is deregulated in aging and human disease. J Clin Invest 125: 1-4, 2015.
34. Guo C, Peng X, Song L, Ying M, Wu Y, Chang R, Li J, Feng D, Zhan $\mathrm{L}$ and Zhan $\mathrm{X}$ : Autophagy promotes malignant migration and invasion via miR-224-5p/BCL2 in pancreatic mucinous cystadenocarcinoma MCC1 cells. Oncol Lett 20: 276, 2020.

35. Cao QH, Liu F, Yang ZL, Fu XH, Yang ZH, Liu Q, Wang L, Wan XB and Fan XJ: Prognostic value of autophagy related proteins ULK1, Beclin 1, ATG3, ATG5, ATG7, ATG9, ATG10, ATG12, LC3B and p62/SQSTM1 in gastric cancer. Am J Transl Res 8: 3831-3847, 2016.

36. Yang L, Peng X, Jin H and Liu J: Long non-coding RNA PVT1 promotes autophagy as ceRNA to target ATG3 by sponging microRNA-365 in hepatocellular carcinoma. Gene 697: 94-102, 2019.

37. Kang Y, Jia Y, Wang Q, Zhao Q, Song M, Ni R and Wang J: Long noncoding RNA KCNQ1OT1 promotes the progression of non-small cell lung cancer via regulating miR-204-5p/ATG3 axis. Onco Targets Ther 12: 10787-10797, 2019.

38. Lin L, Liu X and Lv B: Long non-coding RNA MEG3 promotes autophagy and apoptosis of nasopharyngeal carcinoma cells via PTEN up-regulation by binding to microRNA-21. J Cell Mol Med 25: 61-72, 2021.

39. Li XM, Jiao YY, Luan BH, Wu HX, Wang RR and Zhong J: Long non-coding RNA MIAT promotes gastric cancer proliferation and metastasis via modulating the miR-331-3p/RAB5B pathway. Oncol Lett 20: 355, 2020 